\title{
Dispersion of Surface Waves Supported by Truncated Metasurfaces
}

\author{
M. Camacho, A.P. Hibbins, and J.R. Sambles \\ Dept. of Physics and Astronomy \\ University of Exeter \\ Exeter, United Kingdom
}

\begin{abstract}
The effects introduced in the dispersion of surface waves supported by a metasurface consisting of a negligible thickness perfectly conducting screen perforated with a finite array of slots are studied by means of a very efficient implementation of Galerkin's version of the Method of Moments with a DeltaGap excitation. A family of higher frequency modes each of which is periodically repeated in the momentum spectrum with decreasing amplitude is found in contrast to the single mode dispersion obtained for an infinite array.
\end{abstract}

\section{INTRODUCTION}

Metasurfaces are thin structures that support the propagation of bound surface modes thanks to the reactive behaviour of its constituents [1] and have become a promising candidate for future cheaper and lightweight antennas. Following the work of some of the current authors where it was found that finite arrays support surface waves when excited at the frequency at which extraordinary transmission occurs [2], this paper now extends the study into the excitation of bound surface modes. In contrast to the infinite periodic problem, the analysis of the electric field on the slots can not be reduced to a single unit cell, requiring an efficient implementation of the Method of Moments to keep the CPU time to a minimum. In addition, the lack of symmetry does not allow for the solution of a homogeneous eigenmode problem, as the introduction of an excitation becomes necessary, which following known experimental techniques will be represented by a localized current source [3], [4].

\section{METHOd OF MOMENTS IMPLEMENTATION}

Let us consider the geometry shown in Fig. 1, in which an infinite zero-thickness perfectly conducting screen has been perforated with a finite array of $M=N_{x} \times N_{y}$ slots. In general, the electric field on the slots satisfies the following integral equation

$$
\begin{gathered}
\mathbf{J}^{\mathrm{as}}(x, y)+\sum_{j=1}^{M} \iint_{\eta_{j}} \overline{\mathbf{G}}_{M}\left(x-x^{\prime}, y-y^{\prime}\right) \\
\cdot \mathbf{E}_{t}^{s c}\left(x^{\prime}, y^{\prime}, z=0\right) d x^{\prime} d y^{\prime}=\mathbf{0} \quad(x, y) \in \eta_{i} \\
(i=1, \ldots, M)
\end{gathered}
$$

\author{
R.R. Boix, and F. Medina \\ Dept. of Electronics and Electromagnetism \\ University of Seville \\ Seville, Spain
}

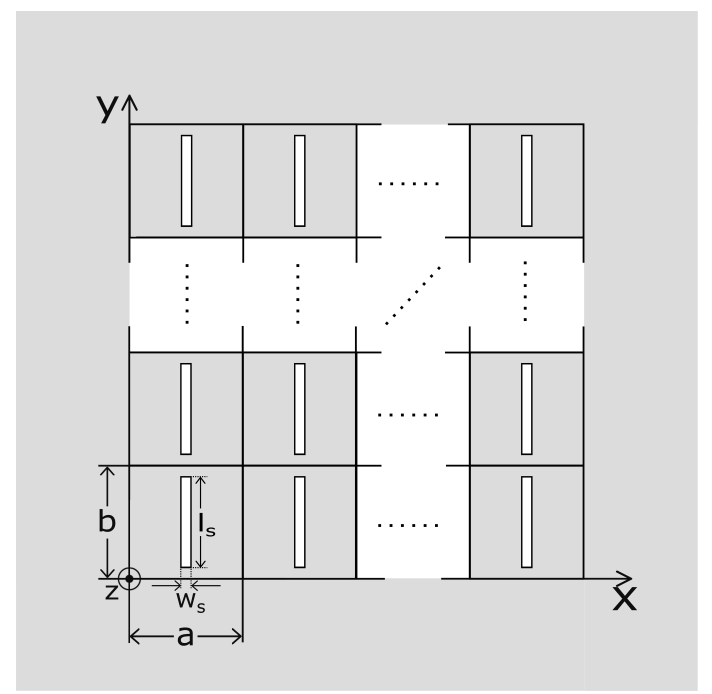

Fig. 1. Truncated periodic array of slots perforated in an infinite negligible thickness perfect conducting screen. The periodicity of the array along the $x$ and $y$ directions are $a$ and $b$ respectively, and $l_{s}$ and $w_{s}$ are the dimensions of the slots.

where $\eta_{i}$ represents the surface of the $i$-th slot and where $\overline{\mathbf{G}}_{M}(x, y)$ is the dyadic Green's function given by

$\overline{\mathbf{G}}_{M}(x, y)=\left(\begin{array}{cc}\left(k_{0}^{2}+\frac{\partial^{2}}{\partial y^{2}}\right) g(x, y) & -\frac{\partial^{2} g(x, y)}{\partial x \partial y} \\ -\frac{\partial^{2} g(x, y)}{\partial x \partial y} & \left(k_{0}^{2}+\frac{\partial^{2}}{\partial x^{2}}\right) g(x, y)\end{array}\right)$

where

$$
g(x, y)=-\frac{\mathrm{je}^{-\mathrm{j} k_{0} \sqrt{x^{2}+y^{2}}}}{\pi k_{0} Z_{0} \sqrt{x^{2}+y^{2}}}
$$

where $k_{0}$ and $Z_{0}$ represent the free space wave vector and impedance respectively. Following previous work, the localized current source will be represented by a spatial dependent current given by $\mathbf{J}^{\text {as }}(x, y) \propto \delta\left(y-y_{c c}\right)$ for $\left(x_{c c}-w_{s} / 2\right)<$ $x<\left(x_{c c}+w_{s} / 2\right)$ and zero otherwise (where $y_{c c}$ and $x_{c c}$ are the $y$ and $x$ coordinates of the center of the central slot respectively) [5]. By applying Galerkin's version of the Method of Moments, the electric field at the slots will be 
expanded as a linear combination of known basis functions given by

$$
\mathbf{E}_{t}^{s c}(x, y, z=0) \approx \sum_{l=1}^{N_{b}} e_{j l} \mathbf{d}_{j l}(x, y) \quad(x, y) \in \eta_{j}
$$

which leads to a system of linear equations for the unknown coefficients $e_{j l}$ given by

$$
\sum_{j=1}^{M} \sum_{l=1}^{N_{b}} \Delta_{i j}^{k l} e_{j l}=p_{i k} \quad\left(i=1, \ldots, M ; k=1, \ldots, N_{b}\right)
$$

where

$$
\begin{gathered}
\Delta_{i j}^{k l}=\iint_{\eta_{i}} \mathbf{d}_{i k}^{*}(x, y) \cdot\left[\iint_{\eta_{j}} \overline{\mathbf{G}}_{M}\left(x-x^{\prime}, y-y^{\prime}\right)\right. \\
\left.\cdot \mathbf{d}_{j l}\left(x^{\prime}, y^{\prime}\right) d x^{\prime} d y^{\prime}\right] d x d y \\
\left(i, j=1, \ldots, M ; k, l=1, \ldots, N_{b}\right)
\end{gathered}
$$

and where

$$
\begin{array}{r}
p_{i k}=-\left(\iint_{\eta_{i}} \mathbf{d}_{i k}^{*}(x, y) \cdot \mathbf{J}^{\mathrm{as}}(x, y) d x d y\right) \\
\left(i=1, \ldots, M ; k=1, \ldots, N_{b}\right)
\end{array}
$$

The matrix coefficient can be efficiently calculated in the spatial domain in terms of the cross-correlations of the basis functions when whole-domain basis functions given by Chebyshev polynomials multiplied by the edge behaviour of the electric field are chosen.

\section{RESULTS}

Once the system of equations is solved, the two-dimensional analytical Fourier transform of the fields is computed for each frequency, obtaining the dispersion diagram of the modes supported by the system. Fig. 2 shows the dispersion obtained for an array of $49 \times 49$ elements (color plot) and that corresponding to an infinite periodic array (white squares). In there, it can be seen how the brightest feature directly compares to the mode found in the infinite array. However, a family of higher frequency modes (not present in the infinite array) are also found. These modes are produced by the lack of periodicity which allows for quantized modes with a different number of minima of electric field along the direction perpendicular to that of propagation. The number and frequency of these modes depends on the number of elements along that direction and therefore reduces for narrower samples. In addition, each of the modes is periodically repeated in the spectral domain with a separation inversely proportional to the size of the array long the direction of propagation. This is similar to the periodic repetition of the modes due to the periodicity in the infinite periodic problem, creating the so-called Brillouin zones.

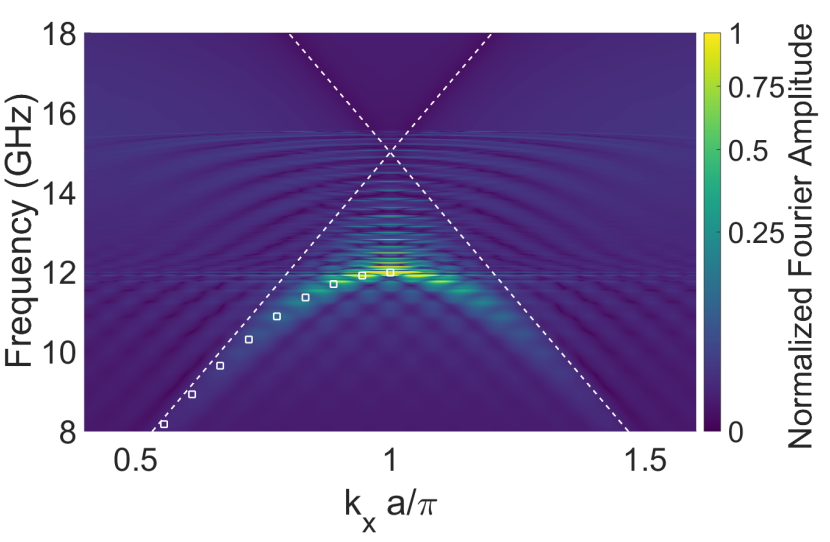

Fig. 2. Electric field Fourier transform predicted by MoM for a range of frequencies for an array of $N_{x}=N_{y}=49$ slots for which $l_{s} / a=0.9$, $w_{s} / a=0.05$ and $a=b=10 \mathrm{~mm}$. It is represented linearly in color as the square root of the absolute value of the Fourier amplitude. The white superposed squares correspond to the dispersion diagram obtained for an infinite periodic array with the same unit cell.

\section{CONCLUSION}

A Method of Moments implementation for the solution of the dispersion of surface waves supported by a finite array of slots has been presented. It has been shown that an array of $49 \times 49$ slots supports a higher number of modes than the corresponding infinite periodic array, showing a family of higher frequency modes associated to the waveguide behaviour of the system along the direction perpendicular to that of propagation. In addition, each of the modes is periodically repeated along the $k$ axis with a periodicity inversely proportional to the size of the sample.

\section{ACKNOWLEDGEMENT}

The authors wish to acknowledge financial support from the Engineering and Physical Sciences Research Council (EPSRC) of the United Kingdom, via the EPSRC Centre for Doctoral Training in Metamaterials (Grant No. EP/L015331/1).

\section{REFERENCES}

[1] S. Maci, G. Minatti, M. Casaletti, and M. Bosiljevac, "Metasurfing: Addressing Waves on Impenetrable Metasurfaces," IEEE Antennas and Wireless Propagation Letters, vol. 10, pp. 1499-1502, 2011. [Online]. Available: http://ieeexplore.ieee.org/document/6127895/

[2] M. Camacho, R. R. Boix, and F. Medina, "Computationally efficient analysis of extraordinary optical transmission through infinite and truncated subwavelength hole arrays," Physical Review E - Statistical, Nonlinear, and Soft Matter Physics, vol. 93, no. 6, p. 063312, jun 2016. [Online]. Available: http://link.aps.org/doi/10.1103/PhysRevE.93.063312

[3] I. R. Hooper, B. Tremain, J. A. Dockrey, and A. P. Hibbins, "Massively Sub-wavelength Guiding of Electromagnetic Waves," Scientific Reports, vol. 4, p. 7495, dec 2014. [Online]. Available: http://www.nature.com/ articles/srep07495

[4] J. A. Dockrey, S. A. R. Horsley, I. R. Hooper, J. R. Sambles, and A. P. Hibbins, "Direct observation of negative-index microwave surface waves," Scientific Reports, vol. 6, no. February, p. 22018, feb 2016. [Online]. Available: http://www.nature.com/articles/srep22018

[5] A. Neto and S. Maci, "Green's function for an infinite slot printed between two homogeneous dielectrics - Part I: Magnetic currents," IEEE Transactions on Antennas and Propagation, vol. 51, no. 7, pp. 1572 1581, 2003. 